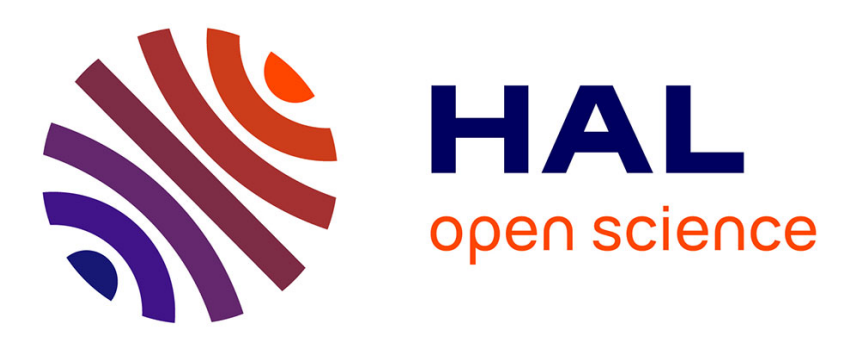

\title{
Observer-Based Control of Position and Tension for an Aerial Robot Tethered to a Moving Platform
}

Marco Tognon, Sanket Suman Dash, Antonio Franchi

\section{To cite this version:}

Marco Tognon, Sanket Suman Dash, Antonio Franchi. Observer-Based Control of Position and Tension for an Aerial Robot Tethered to a Moving Platform. IEEE Robotics and Automation Letters, 2016, 1 (2), pp. 732-737. 10.1109/LRA.2016.2523599 . hal-01285318

\section{HAL Id: hal-01285318 https://hal.science/hal-01285318}

Submitted on 9 Mar 2016

HAL is a multi-disciplinary open access archive for the deposit and dissemination of scientific research documents, whether they are published or not. The documents may come from teaching and research institutions in France or abroad, or from public or private research centers.
L'archive ouverte pluridisciplinaire HAL, est destinée au dépôt et à la diffusion de documents scientifiques de niveau recherche, publiés ou non, émanant des établissements d'enseignement et de recherche français ou étrangers, des laboratoires publics ou privés. 


\title{
Observer-based Control of Position and Tension for an Aerial Robot Tethered to a Moving Platform
}

\author{
Marco Tognon ${ }^{1,2}$, Sanket S. Dash ${ }^{1}$, and Antonio Franchi ${ }^{1,2}$
}

\begin{abstract}
In this paper we address a challenging version of the problem of controlling tethered aerial vehicles (also known as UAV, MAV, and UAS) by considering the aerial robot linked to a generic and independently moving platform. We solve the exact tracking control problem for both the 3D position of the robot (either absolute or with respect to the platform) and the tension along the link. To achieve this goal we prove some fundamental system properties, useful to design a nonlinear controller, such as differential flatness and dynamic feedback linearizability. To close the control loop a set of minimal and standard sensors is proposed. Then we show that it is possible to retrieve the full system state from those sensors by means of nonlinear measurements transformations and a bank of low-dimension estimators based on the nonlinear high gain observer. The ability of the proposed observer-controller method is tested by extensive numerical simulations spanning many non-ideal conditions.
\end{abstract}

Index Terms-Aerial Robotics, Underactuated Robots

\section{INTRODUCTION}

$\mathbf{I}$ $\mathrm{N}$ the area of aerial physical interaction, a novel solution gaining more and more interest consists in the use of a cable to physically connect an aerial vehicle to a fixed base station. Indeed, the use of a cable can overcome the main limitations of aerial robots such as battery life, weak data transfer, low payload, flyability with strong wind and so on.

In this field there are some works [1]-[3] proposing controllers to track/stabilize the $2 \mathrm{D}$ position of the vehicle while preserving the tautness of the cable. Moreover it has been proved that an on-board inertial sensor is enough to estimate the whole state and close the control loop both in the simpler static- [2] and in the more challenging dynamic- [1] cases. Furthermore, the tethered flight solution results really helpful during dangerous tasks. In [4] the tether solution, together with a winch, is used to perform the landing of a small unmanned helicopter, without the use of GPS sensors. In [5] the authors propose a control method to safely land an autonomous helicopter tethered to a moving platform.

In this work we depart from [1]-[3] by considering the more challenging and complete 3D extension of the tethered aerial robot problem. Moreover, the aerial vehicle is linked to an actuated winch, used to wind-up/out the cable, rigidly attached

Manuscript received: August 31, 2015; Revised November 9, 2015; Accepted January 14, 2016.

This paper was recommended for publication by Editor Roberts, Jonathan upon evaluation of the Associate Editor and Reviewers' comments. This work has been partially funded by the European Union's Horizon 2020 research and innovation programme under grant agreement No 644271 AEROARMS.

${ }^{1}$ LAAS-CNRS, 7 Avenue du Colonel Roche, F-31400 Toulouse, France. mtognon@laas.fr, s.dash@enevate.nl, afranchi@laas.fr

${ }^{2}$ Univ de Toulouse, LAAS, F-31400 Toulouse, France

Digital Object Identifier xxxxxxxxxxxxxxxxxxx to a generic platform, instead of a constant-length link attached to a fixed point as in [1]-[3]. The platform is not controlled, i.e., it is generically supposed to translate and rotate in $3 \mathrm{D}$, independently from the aerial robot. Therefore it can represent, e.g., a ground vehicle, a ship or another aerial vehicle.

Furthermore we depart from [4] and [5] by solving the problem of exact tracking of a time-varying desired trajectory of the relative position of the aerial vehicle in the platform frame (or equivalently the absolute position when the platform trajectory is known), instead of just regulating the position to a constant value or to a slowly varying one during the landing maneuver. At the same time we also let the link internal force track any time-varying desired tension profile, while the platform is performing any motion. As shown in previous works the control of the tension is crucial for the aerial vehicle position estimation [1], [6], and to avoid breakages of the link. Finally we close the control loop using only a minimal set of standard sensors thus improving the applicability in real scenarios. A system employing our proposed method can be used for several applications such as inspection, surveillance and autonomous takeoff and landing from moving platforms. To achieve our goals we propose a nonlinear controller based on dynamic feedback linearization, a nonlinear measurement transformation and a nonlinear observer, that are able to track the desired trajectory with zero error in any dynamic conditions. Our design is based on the intrinsic characteristics of the system such as differential flatness, state observability and trajectory feasibility.

The paper is organized as follows. At first we model the system and we formalize the control problem (Sec. II). Then we design a non linear controller (Sec. III) and a nonlinear observer based on a standard sensorial configuration (Sec. IV). Finally the method is validated through numerical simulations (Sec. V) and discussed in some conclusive remarks (Sec. VI).

\section{Modeling, Problem And Metodology}

We consider an aerial vehicle that is tethered by a link (e.g., a cable, a rope, or a chain) to an actuated winch that is, in turn, fixed on a moving platform. The platform moves generically in the 3D space and can be, e.g., a ground vehicle moving on any kind of terrain, a marine vessel, or even another aerial vehicle. Fig. 1 depicts the systems and its main definitions.

Consider a fixed world frame, $\mathscr{F}_{W}$ with axes $\left\{\mathbf{x}_{W}, \mathbf{y}_{W}, \mathbf{z}_{W}\right\}$ and origin $O_{W}$. Two body frames, $\mathscr{F}_{C}$ and $\mathscr{F}_{R}$ with axes $\left\{\mathbf{x}_{C}, \mathbf{y}_{C}, \mathbf{z}_{C}\right\}$ and $\left\{\mathbf{x}_{R}, \mathbf{y}_{R}, \mathbf{z}_{R}\right\}$, and origins $O_{C}$ and $O_{R}$, are rigidly attached to the platform and to the aerial vehicle, respectively. The position of $O_{C}$ in $\mathscr{F}_{W}$ is described by the 


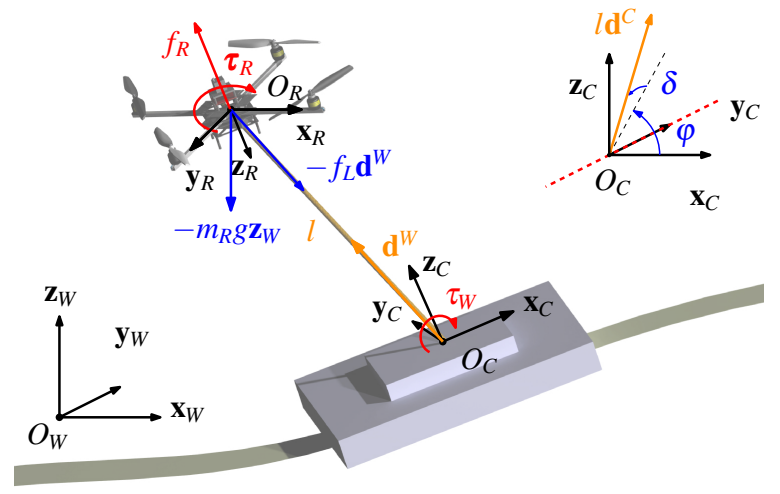

Fig. 1: Left: representation of the system and its main variables. Top right corner: parametrization of the unit vector $\mathbf{d}^{C}$. The red line shows the singularities of the parametrization, avoidable in the planning phase.

vector $\mathbf{p}_{C}^{W}=\left[\begin{array}{lll}x_{C} & y_{C} & z_{C}\end{array}\right]^{T} \in \mathbb{R}^{3}$. Similarly, $O_{R}$ is set on the center of mass $(\mathrm{CoM})$ of the aerial vehicle, whose position in $\mathscr{F}_{W}$ is described by the vector $\mathbf{p}_{R}^{W}=\left[\begin{array}{lll}x_{R} & y_{R} & z_{R}\end{array}\right]^{T} \in \mathbb{R}^{3}$.

The aerial vehicle is a VTOL (Vertical Take-Off and Landing) modeled as a rigid body with mass $m_{R} \in \mathbb{R}^{+}$and positive definite diagonal inertia matrix $\mathbf{J}_{R} \in \mathbb{R}^{3 \times 3}$ (expressed in $\mathscr{F}_{R}$ and relative to $O_{R}$ ). The angular velocity of $\mathscr{F}_{R}$ with respect to $\mathscr{F}_{W}$, expressed in $\mathscr{F}_{R}$, is denoted by $\boldsymbol{\omega}_{R} \in \mathbb{R}^{3}$. The aerial vehicle configuration is fully described by $\mathbf{p}_{R}^{W}$ and by the matrix $\mathbf{R}_{R} \in \mathrm{SO}(3)$ representing the rotation from $\mathscr{F}_{R}$ to $\mathscr{F}_{W}$. The aerial vehicle motion can be controlled acting on four inputs: $f_{R} \in \mathbb{R}$ and $\boldsymbol{\tau}_{R}=\left[\begin{array}{lll}\tau_{R x} & \tau_{R y} & \tau_{R z}\end{array}\right]^{T} \in \mathbb{R}^{3}$, where $f_{R}$ is the magnitude of the thrust force $\mathbf{f}_{R}=-f_{R} \mathbf{z}_{R}$ applied at $O_{R}$ and parallel to $\mathbf{z}_{R}$, and $\tau_{R}$ are the coordinates of the 3D control moment expressed in $\mathscr{F}_{R}$.

The moving platform configuration is described by $\mathbf{p}_{C}^{W}$ and $\mathbf{R}_{C} \in \mathrm{SO}(3)$, representing the rotation from $\mathscr{F}_{C}$ to $\mathscr{F}_{W}$. The angular velocity of $\mathscr{F}_{C}$ with respect to $\mathscr{F}_{W}$, expressed in $\mathscr{F}_{C}$, is denoted by $\boldsymbol{\omega}_{C} \in \mathbb{R}^{3}$. The platform is an independent subsystem whose motion $i$ ) is not influenced by the aerial vehicle dynamics and ii) can only be measured online. In this way, the results can be applied to a broader class of moving platforms including, e.g., human controlled vehicles.

The link connects the aerial vehicle to the moving platform. One end of the link is attached to the aerial vehicle at $O_{R}$ through a passive 3D spherical joint and the other end is attached to the platform at $O_{C}$, through the actuated winch. As in [5], we assume negligible link mass and inertia with respect to the ones of the aerial vehicle and negligible deformations and elasticity. The direction of the link is described by the unit vector $\mathbf{d}^{C} \in \mathrm{S}^{2}$ expressed in $\mathscr{F}_{C}$ thus allowing to express the aerial vehicle position relative to the moving platform. Nevertheless, known $\mathbf{p}_{C}^{W}$ and $\mathbf{R}_{C}$, one can still control $\mathbf{p}_{R}^{W}$ by inverse kinematics. The link length and the intensity of the internal force are denoted by $l \in \mathbb{R}_{\geq 0}$ and $f_{L} \in \mathbb{R}_{>0}$, respectively. The tension of the link is equal to $f_{L}$ when $f_{L}>0$, while it is equal to zero otherwise (slack link). Thus, controlling $f_{L}$ for $f_{L}>0$ is equivalent to control the tension, ensuring the tautness of the link. The unit vector $\mathbf{d}^{C}$ can be parametrized with the elevation angle, $\varphi \in[0,2 \pi]$, and the azimuth angle, $\delta \in\left[-\frac{\pi}{2}, \frac{\pi}{2}\right]$, as $\mathbf{d}^{C}=[\cos \delta \cos \varphi-\sin \delta \cos \delta \sin \varphi]^{T}$, where $\delta$ is the angle between $\mathbf{d}^{C}$ and the vertical plane $\left\{\mathbf{x}_{C}, \mathbf{z}_{C}\right\}$, whereas $\varphi$ is the angle between the projection of $\mathbf{d}^{C}$ on $\left\{\mathbf{x}_{C}, \mathbf{z}_{C}\right\}$ and $\mathbf{x}_{C}$, see Fig. 1. This particular choice lets the singularity of the parametrization correspond to the points along $\mathbf{y}_{C}$, whereas the classical spherical parametrization has the singularity along $\mathbf{z}_{C}$, which corresponds to the common vertical link orientation.

The actuated winch is fixed to the moving platform in the proximity of $O_{C}$ and is used to control $l$ and $f_{L}$ in a coordinated action with the aerial vehicle thrust force. The winch is modeled as a cylinder around which the link is winded up or out and a motor that exerts an input torque $\tau_{W} \in \mathbb{R}$ about the longitudinal axis of the cylinder. The constant rotational inertia and constant radius of the winch are denoted by $J_{W} \in \mathbb{R}^{+}$and $r_{W} \in \mathbb{R}^{+}$, respectively.

Since the link is attached to the aerial vehicle center of mass, the aerial vehicle rotational dynamics is independent from the translational dynamics and it is described by:

$$
\begin{aligned}
\dot{\mathbf{R}}_{R} & =\mathbf{R}_{R} \boldsymbol{\Omega}_{R} \\
\mathbf{J}_{R} \dot{\boldsymbol{\omega}}_{R} & =\mathbf{J}_{R} \boldsymbol{\omega}_{R} \times \boldsymbol{\omega}_{R}+\boldsymbol{\tau}_{R},
\end{aligned}
$$

where $\boldsymbol{\Omega}_{\star}$ is the skew symmetric matrix associated to $\boldsymbol{\omega}_{\star}$. The linear velocity of the aerial vehicle is obtained differentiating $\mathbf{p}_{R}^{W}=\mathbf{p}_{C}^{W}+l \mathbf{R}_{C} \mathbf{d}^{C}$ :

$$
\dot{\mathbf{p}}_{R}^{W}=\mathbf{R}_{C}\left(\dot{\mathbf{p}}_{C}^{C}+l \boldsymbol{\Omega}_{C} \mathbf{d}^{C}+\dot{l}^{C}+l \dot{\mathbf{d}}^{C}\right) .
$$

To derive the dynamic equations of the generalized coordinates $\mathbf{q}=\left[\begin{array}{lll}l & \varphi & \delta\end{array}\right]^{T}$ we use the Newton-Euler approach, solving the balance of the forces acting on $O_{R}$ in $\mathscr{F}_{W}$, and the balance of momenta about the axis of the winch:

$$
\begin{aligned}
m_{R} \ddot{\mathbf{p}}_{R}^{W} & =-f_{L} \mathbf{R}_{C} \mathbf{d}^{C}-f_{R} \mathbf{z}_{R}^{W}-m_{R} g \mathbf{z}_{W}^{W} \\
\bar{J}_{W} \ddot{l} & =\bar{\tau}_{W}+f_{L},
\end{aligned}
$$

where $\bar{J}_{W}=J_{W} / r_{W}^{2}, \bar{\tau}_{W}=\tau_{W} / r_{W}$. The acceleration $\ddot{\mathbf{p}}_{R}^{W}$ is obtained by further differentiating (3), i.e.,

$$
\ddot{\mathbf{p}}_{R}^{W}=\mathbf{R}_{C}\left[\overline{\mathbf{a}}_{x}+\mathbf{J}_{\mathbf{q}} \ddot{\mathbf{q}}\right]
$$

where $\overline{\mathbf{a}}_{x}=\boldsymbol{\Omega}_{C}\left(\dot{\mathbf{p}}_{C}^{C}+l \boldsymbol{\Omega}_{C} \mathbf{d}^{C}+2 \mathbf{J}_{\mathbf{q}} \dot{\mathbf{q}}\right)+\ddot{\mathbf{p}}_{C}^{C}+l \dot{\boldsymbol{\Omega}}_{C} \mathbf{d}^{C}+\dot{\mathbf{J}}_{\mathbf{q}} \dot{\mathbf{q}}$ and

$$
\mathbf{J}_{\mathbf{q}}=\left[\begin{array}{ccc}
\cos \delta \cos \varphi & -l \cos \delta \sin \varphi & -l \cos \varphi \sin \delta \\
-\sin \delta & 0 & -l \cos \delta \\
\cos \delta \sin \varphi & l \cos \delta \cos \varphi & -l \sin \delta \sin \varphi
\end{array}\right] .
$$

Replacing (6) into (4) and after some algebra we get

$$
m_{R} \mathbf{J}_{\mathbf{q}} \ddot{\mathbf{q}}+f_{L} \mathbf{d}^{C}=-\underbrace{m_{R} \overline{\mathbf{a}}_{x}}_{\mathbf{a}_{x}}-\underbrace{m_{R} g \mathbf{R}_{C}^{T} \mathbf{e}_{3}}_{\mathbf{a}_{g}}-\underbrace{f_{R} \mathbf{R}_{C}^{T} \mathbf{R}_{R} \mathbf{e}_{3}}_{\mathbf{a}_{f_{R}}},
$$

Finally, gathering (7) and (5) we obtain a square system

$$
\underbrace{\left[\begin{array}{cc}
m_{R} \mathbf{J}_{\mathbf{q}} & \mathbf{d}^{C} \\
\mathbf{J}_{W \mathbf{q}} & -1
\end{array}\right]}_{\mathbf{W}}\left[\begin{array}{c}
\ddot{\mathbf{q}} \\
f_{L}
\end{array}\right]=\underbrace{\left[\begin{array}{c}
-\mathbf{a}_{x}-\mathbf{a}_{g} \\
0
\end{array}\right]}_{\mathbf{a}}+\underbrace{\left[\begin{array}{c}
-\mathbf{a}_{f_{R}} \\
\bar{\tau}_{W}
\end{array}\right]}_{\mathbf{a}_{\mathbf{u}}}
$$

where $\mathbf{J}_{W \mathbf{q}}=\left[\begin{array}{lll}\bar{J}_{W} & 0 & 0\end{array}\right] \in \mathbb{R}^{1 \times 3}$ and $\mathbf{W} \in \mathbb{R}^{4 \times 4}$ is invertible if and only if $l \neq 0$ and $\delta \neq \pm \pi / 2$, that correspond to the singularities of the pseudo-spherical coordinates of $O_{R}$. Inverting equation (8) out of these singularities, we obtain

$$
\begin{aligned}
\ddot{\mathbf{q}} & =\left[\begin{array}{ll}
\mathbf{I}_{3} & \mathbf{0}_{3 \times 1}
\end{array}\right] \mathbf{W}^{-1}\left(\mathbf{a}+\mathbf{a}_{\mathbf{u}}\right)=\sigma\left(\mathbf{x}, \mathbf{X}_{C}^{2}, \mathbf{u}\right) \\
f_{L} & =\left[\begin{array}{ll}
\mathbf{0}_{1 \times 3} & 1
\end{array}\right] \mathbf{W}^{-1}\left(\mathbf{a}+\mathbf{a}_{\mathbf{u}}\right)
\end{aligned}
$$


where $\mathbf{x}=\left(\mathbf{q}, \dot{\mathbf{q}}, \mathbf{R}_{R}, \boldsymbol{\omega}_{R}\right) \quad$ is the system state, $\mathbf{u}=\left[\begin{array}{lll}f_{R} & \boldsymbol{\tau}_{R}^{T} & \bar{\tau}_{W}\end{array}\right]^{T}=\left[\begin{array}{lllll}u_{1} & u_{2} & u_{3} & u_{4} & u_{5}\end{array}\right]^{T}$ is the vector of inputs and $\mathbf{X}_{C}^{j}=\left(\mathbf{x}_{C}^{0}, \mathbf{x}_{C}^{1}, \ldots, \mathbf{x}_{C}^{j}\right)$ for $j \in \mathbb{N}^{+}$, with $\mathbf{x}_{C}^{i}=\left(\mathbf{p}_{C}^{C(i)}, \boldsymbol{\omega}_{C}^{(i-1)}\right)$ for $i=1,2, \ldots$ and $\mathbf{x}_{C}^{0}=\left(\mathbf{p}_{C}^{W}, \mathbf{R}_{C}\right)$, gathers the terms related to the motion of the platform. Equations (1), (2) and (9) provide a complete description of the system dynamics.

\section{Problem, Objectives, and Results}

In this work we address a three-objective problem. The first objective is to provide a control strategy to let $\mathbf{p}_{R}^{C}$ and $f_{L}$ (or, equivalently, $\mathbf{q}$ and $f_{L}$ ) exactly track any sufficiently smooth desired trajectory $\mathbf{p}_{R}^{C d}$ and $f_{L}^{d}$ (or, equivalently, $\mathbf{q}^{d}$ and $f_{L}^{d}$ ). The second objective is to provide a method to know in advance i) how the full state of the system behaves when tracking a desired output trajectory and ii) which are the nominal inputs required to track it. The last objective is to provide the mathematical tools to implement the control strategy in reality using a minimal set of typically available onboard sensors.

To achieve the previous objectives we first show the exact linearizability and the differential flatness of the system designing a nonlinear controller. We then design a nonlinear observer for the full state that uses a minimal set of standard sensors. Finally we check through realistic simulations the practicability of the proposed method in non-ideal scenarios.

\section{DynAmic Decoupling CONTROL}

In this work we want to control the four outputs corresponding to $l, \varphi, \delta$ and $f_{L}$, using the control input $\mathbf{u}$. Notice that the outputs of interest are independent from $\tau_{R z}$. Intuitively, only $\bar{\tau}_{W}$ and $\mathbf{f}_{R}$ play a role in the control of the outputs, see (8), and they are not affected by $\tau_{R z}$. Indeed $\mathbf{f}_{R}$ is not influenced by rotations along $\mathbf{z}_{R}$ and therefore not even by the torque $\tau_{R z}$ acting about it. Then it is necessary to complete the set of outputs with a quantity dynamically dependent on $\tau_{R z}$ to have a well-posed tracking problem. It is sufficient to consider any generic parametrization $\boldsymbol{\eta}=\left[\begin{array}{lll}\eta_{1} & \eta_{3} \eta_{3}\end{array}\right]^{T} \in \mathbb{R}^{3}$ of $\mathbf{R}_{R}$, such that $\mathbf{R}_{R}=\mathbf{R}_{R}(\boldsymbol{\eta})$ and $\dot{\boldsymbol{\eta}}=\mathbf{T}_{\boldsymbol{\eta}} \boldsymbol{\omega}_{R}$ where $\mathbf{T}_{\boldsymbol{\eta}}(\boldsymbol{\eta}) \in \mathbb{R}^{3 \times 3}$ is given by the particular parametrization. From (2) the dynamics of $\boldsymbol{\eta}$ is

$$
\begin{aligned}
\ddot{\boldsymbol{\eta}} & =\dot{\mathbf{T}}_{\boldsymbol{\eta}} \boldsymbol{\omega}_{R}+\mathbf{T}_{\boldsymbol{\eta}} \mathbf{J}_{R}^{-1}\left(\mathbf{J}_{R} \boldsymbol{\omega}_{R} \times \boldsymbol{\omega}_{R}\right)+\left[\begin{array}{lll}
\mathbf{0}_{3 \times 1} & \mathbf{T}_{\boldsymbol{\eta}} \mathbf{J}_{R}^{-1} & \mathbf{0}_{3 \times 1}
\end{array}\right] \mathbf{u} \\
& =\mathbf{b}_{\boldsymbol{\eta}}(\boldsymbol{\eta}, \dot{\boldsymbol{\eta}})+\mathbf{E}_{\boldsymbol{\eta}}(\boldsymbol{\eta}) \mathbf{u} .
\end{aligned}
$$

Then we consider as output of the system the variables $\mathbf{y}=$ $\left[\begin{array}{lllll}y_{1} & y_{2} & y_{3} & y_{4} & y_{5}\end{array}\right]^{T}=\left[\begin{array}{lllll}l & \varphi & \delta & f_{L} & \eta_{i}\end{array}\right]^{T} \in \mathbb{R}^{5}$ where $\eta_{i}$ is any entry of $\boldsymbol{\eta}$ such that, in the domain of interest, it holds

$$
e_{\eta_{i}}=\frac{\partial \ddot{\eta}_{i}}{\partial \tau_{R z}}=\mathbf{e}_{i}^{T} \mathbf{T}_{\boldsymbol{\eta}} \mathbf{J}_{R}^{-1} \mathbf{e}_{3} \neq 0
$$

For example, taking $\boldsymbol{\eta}=\left[\begin{array}{lll}\phi & \theta & \psi\end{array}\right]^{T}$ as the classical Roll-PitchYaw parametrization of $\mathbf{R}_{R}$ and $\eta_{i}=\psi$, we have that

$$
\mathbf{T}_{\boldsymbol{\eta}}(\boldsymbol{\eta})=\left[\begin{array}{ccc}
1 & \sin \phi \tan \theta & \cos \phi \tan \theta \\
0 & \cos \phi & -\sin \phi \\
0 \sin \phi \sec \theta & \cos \phi \sec \theta
\end{array}\right] \text { and } e_{\eta_{i}}=\frac{1}{J_{R 33}} \cos \phi \sec \theta
$$

where $J_{R k m}$ with $k, m \in\{1,2,3\}$ corresponds to the element of the matrix $\mathbf{J}_{R}$ in position $k, m$. Notice that for this choice (11) holds always except for $\phi=\pi / 2$ and $\theta=\pi / 2$.

Applying the feedback linearization technique, recalling equations (8) and (10), we immediately see that $\left(y_{1}, y_{2}, y_{3}\right)$ have to be differentiated twice until $f_{R}$ and $\bar{\tau}_{W}$ appear, $y_{4}$ directly depends on the same two inputs and $y_{5}$ has to be differentiated twice until $\tau_{R}$ appears. Defining $\overline{\mathbf{y}}_{1}=\left[\begin{array}{llll}\ddot{y}_{1} & \ddot{y}_{2} & \ddot{y}_{3} & y_{4}\end{array}\right]^{T}$ and rearranging (8) and (10), we can write

$$
\left[\begin{array}{c}
\overline{\mathbf{y}}_{1} \\
\ddot{y}_{5}
\end{array}\right]=\left[\begin{array}{c}
\overline{\mathbf{W}} \mathbf{a} \\
b_{\eta_{i}}
\end{array}\right]+\left[\begin{array}{c}
\overline{\mathbf{W}} \mathbf{U} \\
\mathbf{e}_{\eta_{i}}
\end{array}\right] \mathbf{u}=\mathbf{b}\left(\mathbf{x}, \mathbf{X}_{C}^{2}\right)+\mathbf{E}\left(\mathbf{x}, \mathbf{X}_{C}^{0}\right) \mathbf{u},
$$

where $\overline{\mathbf{W}}=\mathbf{W}^{-1}, b_{\eta_{i}}=\mathbf{e}_{i}^{T} \mathbf{b}_{\boldsymbol{\eta}}, \mathbf{e}_{\eta_{i}}=\mathbf{e}_{i}^{T} \mathbf{E}_{\boldsymbol{\eta}}$ and

$$
\mathbf{U}=\left[\begin{array}{ccc}
-\mathbf{R}_{C}^{T} \mathbf{R}_{R} \mathbf{e}_{3} & \mathbf{0}_{3 \times 3} & 0 \\
0 & \mathbf{0}_{1 \times 3} & 1
\end{array}\right]_{4 \times 5}, \mathbf{E}=\left[\begin{array}{ccc}
-\mathbf{R}_{C}^{T} \mathbf{R}_{R} \mathbf{e}_{3} & \mathbf{0}_{3 \times 3} & \mathbf{0}_{3 \times 1} \\
0 & \mathbf{0}_{1 \times 3} & 1 \\
0 & \mathbf{e}_{i}^{T} \mathbf{T}_{\boldsymbol{\eta}} \mathbf{J}_{R}^{-1} & 0
\end{array}\right]_{5 \times 5},
$$

where the vector $\mathbf{b}\left(\mathbf{x}, \mathbf{X}_{C}^{2}\right)$ gathers all the terms that do not depend on the inputs. The decoupling matrix $\mathbf{E}$, rearranging the rows, is clearly singular. To obtain a full rank matrix we insert a dynamic compensator considering as new inputs $\overline{\mathbf{u}}=$ $\left[\begin{array}{lllll}\ddot{u}_{1} & u_{2} & u_{3} & u_{4} & \ddot{u}_{5}\end{array}\right]^{T}$, where $\ddot{u}_{1}$ and $\ddot{u}_{5}$ are the second derivative of $f_{R}$ and $\bar{\tau}_{W}$, respectively. Under this definition $\overline{\mathbf{y}}_{1}$ and $y_{5}$ have to be differentiated twice to see the new inputs appear:

$$
\left[\begin{array}{c}
\ddot{\mathbf{y}}_{1} \\
\ddot{y}_{5}
\end{array}\right]=\left[\begin{array}{c}
\ddot{\mathbf{W}}\left(\mathbf{a}+\mathbf{a}_{\mathbf{u}}\right)+2 \dot{\overline{\mathbf{W}}}\left(\dot{\mathbf{a}}+\dot{\mathbf{a}}_{\mathbf{u}}\right)+\overline{\mathbf{W}}\left(\ddot{\mathbf{a}}+\ddot{\mathbf{a}}_{\mathbf{u}}\right) \\
b_{i}+\mathbf{e}_{\eta_{i}} \overline{\mathbf{u}}
\end{array}\right],
$$

where $\ddot{\mathbf{a}}_{\mathbf{u}}$, after replacing the system dynamics, results:

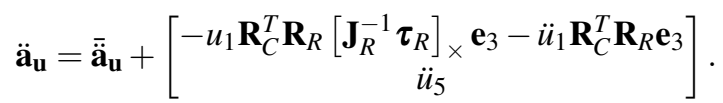

Since $\mathbf{J}_{R}$ is diagonal, i.e., $J_{R k m}=0$ for $k \neq m$ and $k, m \in$ $\{1,2,3\}$, writing the skew symmetric matrix relative to $\mathbf{J}_{R}^{-1} \boldsymbol{\tau}_{R}$ and doing some algebra we obtain

$$
\left[\mathbf{J}_{R}^{-1} \boldsymbol{\tau}_{R}\right]_{\times} \mathbf{e}_{3}=\left[\begin{array}{lll}
-\frac{\mathbf{e}_{2}}{J_{R 11}} & \frac{\mathbf{e}_{1}}{J_{R 22}} & \mathbf{0}_{3 \times 1}
\end{array}\right] \boldsymbol{\tau}_{R} .
$$

Replacing equations (15) and (14) into (13) we obtain

$$
\left[\begin{array}{c}
\ddot{\mathbf{y}}_{1} \\
\ddot{y}_{2}
\end{array}\right]=\underbrace{\left[\begin{array}{c}
\left.\ddot{\mathbf{W}}\left(\mathbf{a}+\mathbf{a}_{\mathbf{u}}\right)+2 \dot{\overline{\mathbf{W}}}\left(\dot{\mathbf{a}}+\dot{\mathbf{a}}_{\mathbf{u}}\right)+\overline{\mathbf{W}}\left(\ddot{\mathbf{a}}+\overline{\mathbf{a}}_{\mathbf{u}}\right)\right] \\
b_{i}
\end{array}\right]}_{\overline{\mathbf{b}}\left(\overline{\mathbf{x}}, \mathbf{X}_{C}^{4}\right)}+\underbrace{\left[\begin{array}{c}
\overline{\mathbf{W}} \overline{\mathbf{U}} \\
\mathbf{e}_{\eta_{i}}
\end{array}\right]}_{\overline{\mathbf{E}}\left(\overline{\mathbf{x}}, \mathbf{X}_{C}^{0}\right)} \overline{\mathbf{u}},
$$

where $\overline{\mathbf{U}}=\left[\begin{array}{cc}-\mathbf{R}_{C}^{T} \mathbf{R}_{R} \mathbf{T} & \mathbf{0}_{3 \times 1} \\ \mathbf{0}_{1 \times 4} & 1\end{array}\right], \overline{\mathbf{x}}=\left(\mathbf{q}, \dot{\mathbf{q}}, \mathbf{R}_{R}, \boldsymbol{\omega}_{R}, f_{R}, \dot{f}_{R}, \bar{\tau}_{W}, \dot{\bar{\tau}}_{W}\right)$ is the extended state, and $\mathbf{T}=\left[\mathbf{e}_{3}-\frac{u_{1}}{J_{R 11}} \mathbf{e}_{2} \frac{u_{1}}{J_{R 22}} \mathbf{e}_{1} \mathbf{0}_{3 \times 1}\right] \in \mathbb{R}^{3 \times 4}$. Changing the order of the inputs as in $\tilde{\mathbf{u}}=\left[\begin{array}{lllll}u_{1} & u_{2} & u_{3} & u_{5} & u_{4}\end{array}\right]^{T}$, the decoupling matrix becomes $\tilde{\mathbf{E}}=\left[\begin{array}{cc}\tilde{\mathbf{E}}_{1} & \mathbf{0}_{1 \times 3} \\ \tilde{\mathbf{e}}_{3} & e_{\eta_{i}}\end{array}\right]$, where $\tilde{\mathbf{E}}_{1}=$ $\overline{\mathbf{W}}\left[\begin{array}{cc}\tilde{\mathbf{U}}_{1} & \mathbf{0}_{3 \times 1} \\ \mathbf{0}_{1 \times 3} & 1\end{array}\right], \quad \tilde{\mathbf{e}}_{3}=\left[\begin{array}{llll}0 & \mathbf{e}_{i}^{T} \mathbf{T}_{\boldsymbol{\eta}} \mathbf{J}_{R}^{-1} \mathbf{e}_{1} & \mathbf{e}_{i}^{T} \mathbf{T}_{\boldsymbol{\eta}} \mathbf{J}_{R}^{-1} \mathbf{e}_{2} & 0\end{array}\right]$, $\tilde{\mathbf{U}}_{1}=-\mathbf{R}_{C}^{T} \mathbf{R}_{R}\left[\begin{array}{lll}\mathbf{e}_{3} & -\frac{u_{1}}{J_{R 11}} \mathbf{e}_{2} & \frac{u_{1}}{J_{R 22}} \mathbf{e}_{1}\end{array}\right]=-\mathbf{R}_{C}^{T} \mathbf{R}_{R} \tilde{\mathbf{T}}$.

The original decoupling matrix $\tilde{\mathbf{E}}$ is invertible if $\tilde{\mathbf{E}}$ is invertible, or equivalently, due to its triangular form, if $\tilde{\mathbf{E}}_{1}$ is invertible and $e_{\eta_{i}}$ is nonzero. Since the matrices $\mathbf{R}_{C}, \mathbf{R}_{R}$ and $\overline{\mathbf{W}}$ are always full rank (except in the model singularities, i.e., $l=0$ and $\delta= \pm \pi / 2$ ), then $\tilde{\mathbf{U}}_{1}$ is invertible whenever $\tilde{\mathbf{T}}$ is full rank, i.e., if $u_{1} \neq 0$, indeed $\operatorname{det}(\tilde{\mathbf{T}})=u_{1}^{2} /\left(J_{R 11} J_{R 22}\right)$.

In the cases in which the thrust $u_{1}$ is not zero and with the opportune parametrization of $\mathbf{R}_{R}$, using the control law

$$
\overline{\mathbf{u}}=\overline{\mathbf{E}}\left(\overline{\mathbf{x}}, \mathbf{X}_{C}^{0}\right)^{-1}\left[-\overline{\mathbf{b}}\left(\overline{\mathbf{x}}, \mathbf{X}_{C}^{4}\right)+\mathbf{v}\right]
$$

where $\mathbf{v}=\left[\begin{array}{lllll}v_{1} & v_{2} & v_{3} & v_{4} & v_{5}\end{array}\right]^{T} \in \mathbb{R}^{5}$ are virtual inputs, we obtain

$$
y_{1}^{(4)}=v_{1}, \quad y_{2}^{(4)}=v_{2}, \quad y_{3}^{(4)}=v_{3}, \quad y_{4}^{(2)}=v_{4}, y_{5}^{(2)}=v_{5} \text {. }
$$




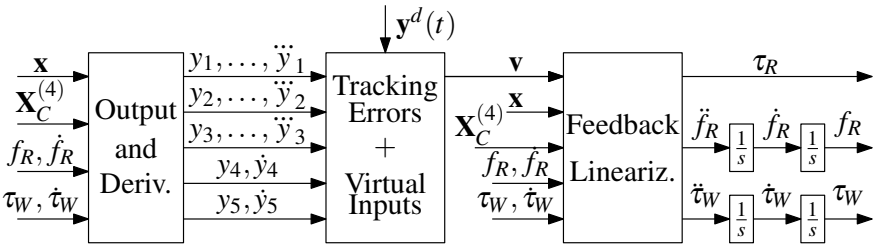

Fig. 2: Graphic representation of the control strategy.

Furthermore, the total relative degree with respect to $\mathbf{y}$ is $r=16$ that corresponds to the dimension of the extended state $\overline{\mathbf{x}}$ that is $\bar{n}=16$. Indeed it is composed by $\mathbf{x}$ (of dimension 12) plus the four states of the dynamic compensator. Therefore the system is exactly dynamic feedback linearizable and the linearized system (17) does not have an internal dynamics [7].

The tracking of any given desired trajectories, $y_{i}^{d}(t) \in C^{3}$ for $i=1,2,3$ and $y_{j}^{d}(t) \in C^{1}$ for $j=4,5$ can be achieved applying any linear control technique to the equivalent linear system (17), as depicted in Fig. 2. E.g., it is sufficient to use as outer loop a simple controller based on the pole placing technique. Setting the virtual control inputs as

$$
v_{i}=y_{i}^{d(4)}+\mathbf{k}_{i}^{T} \boldsymbol{\xi}_{i}, \quad v_{j}=y_{j}^{d(2)}+\mathbf{k}_{j}^{T} \boldsymbol{\xi}_{j},
$$

where $\boldsymbol{\xi}_{i}=\left[\begin{array}{llll}\xi_{i}^{(3)} & \ddot{\xi}_{i} & \dot{\xi}_{i} & \xi_{i}\end{array}\right]^{T} \in \mathbb{R}^{4}, \boldsymbol{\xi}_{j}=\left[\begin{array}{ll}\dot{\xi}_{j} & \xi_{j}\end{array}\right]^{T} \in \mathbb{R}^{2}, \xi_{i}=$ $y_{i}^{d}-y_{i}$ and $\xi_{j}=y_{j}^{d}-y_{j}$ are the tracking errors, one can set the poles of the error dynamics through the gains $\mathbf{k}_{i} \in \mathbb{R}_{>0}^{4}$ and $\mathbf{k}_{j} \in \mathbb{R}_{>0}^{2}$, for $i=1,2,3$ and $j=4,5$, to obtain a sufficiently fast exponentially tracking of the desired trajectories.

All the previous results are summarized in the following

Proposition 1. For the analyzed system it exists at least one parametrization $\boldsymbol{\eta}$ of $\mathbf{R}_{R}$ and one of its elements $\eta_{i}$ such that $\mathbf{y}=\left[\begin{array}{lllll}l & \varphi & \delta & f_{L} & \eta_{i}\end{array}\right]^{T}$ is an exact feedback linearizing output for each state, except for the zero thrust case. Furthermore, considering as input $\overline{\mathbf{u}}=\left[\begin{array}{lllll}\ddot{u}_{1} & u_{2} & u_{3} & u_{4} & \ddot{u}_{5}\end{array}\right]^{T}$, the control laws (16) and (18) exponentially steer $\mathbf{y}$ along the desired trajectories $y_{i}^{d}(t) \in C^{3}$ for $i=1,2,3$, and $y_{j}^{d}(t) \in C^{1}$ for $j=4,5$.

Remark 1. In order to implement the exact tracking control law (16)-(18) the only needed quantities are

- the desired output trajectory and its derivatives $y_{i}^{d}, \dot{y}_{i}^{d}, \ddot{y}_{i}^{d}, \dddot{y}_{i}^{d}, \dddot{y}_{i}^{d}$ for $i=1,2,3$, and $y_{j}^{d}, \dot{y}_{j}^{d}, \ddot{y}_{j}^{d}$ for $j=4,5$

- a measurement of system state, i.e., $\mathbf{x}=\left(\mathbf{q}, \dot{\mathbf{q}}, \mathbf{R}_{R}, \boldsymbol{\omega}_{R}\right)$

- the internal state of the compensators $f_{R}, \dot{f}_{R}, \bar{\tau}_{W}, \dot{\bar{\tau}}_{W}$

- the position and orientation of the moving platform and their derivatives $\mathbf{X}_{C}^{4}$.

From the theory we know that an exactly dynamical feedback linearizing output is also a flat output on an open and dense set of the state space [8]. Therefore the following proposition holds.

Proposition 2. A system made by an underactuated aerial vehicle tethered by a link to an actuated winch fixed on a moving platform is differentially flat with respect to $\mathbf{y}=\left(l, \varphi, \delta, f_{L}, \eta_{i}\right)$ where $\eta_{i}$ is, e.g., the yaw angle of $\mathbf{R}_{R}$.

The flatness lets us to compute the expected state and the nominal inputs required by the desired trajectories [9].

\begin{tabular}{ccccc}
\hline$\#$ & Type & Position & Reference & Measurement \\
\hline $\mathbf{w}_{1}$ & - & $O_{C}$ & $\mathscr{F}_{W}$ & $\mathbf{X}_{C}^{4}$ \\
$w_{2}$ & absolute encoder & $O_{C}$ & $\mathscr{F}_{C}$ & $\vartheta_{W} \approx l$ \\
$w_{3}$ & absolute encoder & $O_{C}$ & $\mathscr{F}_{C}$ & $\varphi$ \\
$w_{4}$ & absolute encoder & $O_{C}$ & $\mathscr{F}_{C}$ & $\delta$ \\
$\mathbf{w}_{5}$ & accelerometer & $O_{R}$ & $\mathscr{F}_{R}$ & $\mathbf{R}_{R}^{T}\left(\ddot{\mathbf{p}}_{R}^{W}+g \mathbf{e}_{3}\right)$ \\
$\mathbf{w}_{6}$ & gyroscope & $O_{R}$ & $\mathscr{F}_{R}$ & $\boldsymbol{\omega}_{R}$ \\
$\mathbf{w}_{7}$ & magnetometer & $O_{R}$ & $\mathscr{F}_{R}$ & $\mathbf{R}_{R}^{T} \mathbf{h}^{W}$ \\
\hline
\end{tabular}

TABLE I: List of sensors.

\section{State Observer}

Assuming that $\mathbf{w}_{1}=\mathbf{X}_{C}^{4}$ is a priori known or it is estimated/measured on-line by a set of sensors, then only the knowledge of $\mathbf{x}$ is needed to close the control loop. In this section we shall demonstrate that the tautness of the cable (i.e., $f_{L}>0$ ) allows to retrieve $\mathbf{x}$ from the standard set of sensors summarized in Tab. I, avoiding the use of sensors as cameras or GPS. First, we assume to have an encoder that gives the absolute rotation of the winch, i.e., the length of the link, $w_{2}=r_{W} \vartheta_{W}=l$. Using a gimbal like mechanism based on encoders (like the one in [4]), we measure the direction of the link, i.e., $w_{3}=\varphi$ and $w_{4}=\delta$. Then, we assume that the aerial vehicle is equipped with standard i) 3 -axis accelerometer that measures the specific acceleration of $O_{R}$ in $\mathscr{F}_{R}$, i.e.:

$$
\mathbf{w}_{5}=\mathbf{R}_{R}^{T}\left(\ddot{\mathbf{p}}_{R}^{W}+g \mathbf{e}_{3}\right),
$$

ii) gyroscope that measures the angular velocity of $\mathscr{F}_{R}$ with respect to $\mathscr{F}_{W}$, expressed in $\mathscr{F}_{R}$, i.e., $\mathbf{w}_{6}=\boldsymbol{\omega}_{R}$, iii) and magnetometer mounted in $O_{R}$ and aligned along the axes of $\mathscr{F}_{R}$ that measures the known unit vector $\mathbf{h}^{W} \in \mathrm{S}^{2}$ describing the magnetic field direction expressed in $\mathscr{F}_{R}: \mathbf{w}_{7}=\mathbf{R}_{R}^{T} \mathbf{h}^{W}$.

Using this sensorial configuration, part of the state is already measured, nevertheless it remains to estimate $\mathbf{R}_{R}$ and $\dot{\mathbf{q}}$.

First we show that, using the accelerometer and the magnetometer, we know how $\mathbf{d}^{W}$ and $\mathbf{h}^{W}$ expressed in $\mathscr{F}_{W}$ are mapped into $\mathscr{F}_{R}$ by $\mathbf{R}_{R}$. Then, creating an orthonormal basis from $\mathbf{d}^{W}$ and $\mathbf{h}^{W}$, and another one from $\mathbf{d}^{R}$ and $\mathbf{h}^{R}$, we can retrieve $\mathbf{R}_{R}$. Substituting (4) into (19), we obtain

$$
f_{L} \mathbf{R}_{R}^{T} \mathbf{R}_{C}\left(\mathbf{w}_{1}\right) \mathbf{d}^{C}\left(w_{3}, w_{4}\right)=-m_{R} \mathbf{w}_{5}-f_{R} \mathbf{e}_{3} .
$$

Define $w_{8}=\left\|-m_{R} \mathbf{w}_{5}-f_{R} \mathbf{e}_{3}\right\|$ and notice that, since the controller guarantees a taut link then $f_{L}>0$ and, in particular, $f_{L}=w_{8}$. Defining $\mathbf{s}_{1}^{R}=\left(-m_{R} \mathbf{w}_{5}-f_{R} \mathbf{e}_{3}\right) / w_{8}$ and $\mathbf{s}_{1}^{W}=\mathbf{R}_{C}\left(\mathbf{w}_{1}\right) \mathbf{d}^{C}\left(w_{3}, w_{4}\right)$ we have that $\mathbf{R}_{R} \mathbf{s}_{1}^{R}=\mathbf{s}_{1}^{W}$. Under the assumption that $\mathbf{s}_{1}^{R}$ and $\mathbf{w}_{7}$ are not parallel, let us define $\mathbf{s}_{2}^{R}=\left(\mathbf{s}_{1}^{R} \times \mathbf{w}_{7}\right) /\left\|\mathbf{s}_{1}^{R} \times \mathbf{w}_{7}\right\|$ and $\mathbf{s}_{3}^{R}=\mathbf{s}_{1}^{R} \times \mathbf{s}_{2}^{R}$. We then obtain

$$
\begin{aligned}
& \mathbf{R}_{R} \mathbf{s}_{2}^{R}=\mathbf{R}_{R}\left(\mathbf{s}_{1}^{R} \times \mathbf{w}_{7}\right) /\left\|\mathbf{s}_{1}^{R} \times \mathbf{w}_{7}\right\|=\left(\mathbf{s}_{1}^{W} \times \mathbf{h}^{W}\right) /\left\|\mathbf{s}_{1}^{W} \times \mathbf{h}^{W}\right\|=\mathbf{s}_{2}^{W} \\
& \mathbf{R}_{R} \mathbf{s}_{3}^{R}=\mathbf{R}_{R}\left(\mathbf{s}_{1}^{R} \times \mathbf{s}_{2}^{R}\right)=\mathbf{s}_{1}^{W} \times \mathbf{s}_{2}^{W}=\mathbf{s}_{3}^{W},
\end{aligned}
$$

where $\left\{\mathbf{s}_{1}^{R}, \mathbf{s}_{2}^{R}, \mathbf{s}_{3}^{R}\right\}$ is an orthonormal basis and $\mathbf{S}^{R}=$ $\left[\begin{array}{lll}\mathbf{s}_{1}^{R} & \mathbf{s}_{2}^{R} & \mathbf{s}_{3}^{R}\end{array}\right] \in \mathrm{SO}(3)$. Defining $\mathbf{S}^{W}=\left[\begin{array}{llll}\mathbf{S}_{1}^{W} & \mathbf{s}_{2}^{W} & \mathbf{s}_{3}^{W}\end{array}\right]$, we obtain

$$
\mathbf{R}_{R}=\mathbf{S}^{W} \mathbf{S}^{R^{T}}=\mathbf{W}_{R}\left(\mathbf{w}_{1}, w_{3}, w_{4}, \mathbf{w}_{5}, \mathbf{w}_{7}\right) .
$$

Notice that we can find $\mathbf{R}_{R}$ only if $\mathbf{d}^{W}$ and $\mathbf{h}^{W}$ are not parallel (otherwise $\mathbf{s}_{1}^{W} \times \mathbf{h}^{W}=\mathbf{0}_{3}$ ) and if $f_{L} \neq 0$. Indeed, if $f_{L}=0$ (slack link) the aerial vehicle and the rest of the system are decoupled, which makes it impossible to estimate the 
attitude of the vehicle in a direct way. Nevertheless, this is not a practical issue since the proposed controller guarantees any positive tension ${ }^{1}$. In the presence of noisy measurements one can exploit $\mathbf{W}_{R}$ and $\mathbf{w}_{6}$ by designing a filter to obtain a better estimation of $\mathbf{R}_{R}$ and $\boldsymbol{\omega}_{R}$ in a way similar, e.g., to [11].

After having shown how to estimate $\mathbf{R}_{R}$, it remains to estimate $\dot{\mathbf{q}}$. Defining $\mathbf{z}=\left[\begin{array}{llllll}l & \dot{l} & \varphi & \dot{\varphi} & \delta & \dot{\delta}\end{array}\right] \in \mathbb{R}^{6}$ we can write its dynamics (see (9)) and the respective measurements as

$$
\dot{\mathbf{z}}=\mathbf{A z}+\mathbf{B} \boldsymbol{\sigma}\left(\mathbf{z}, \mathbf{u}, \mathbf{R}_{R}, \mathbf{X}_{C}^{2}\right), \quad \mathbf{w}_{\mathbf{z}}=\left[\begin{array}{lll}
w_{2} & w_{3} & w_{4}
\end{array}\right]^{T}=\mathbf{C z},
$$

where $\mathbf{A}=\operatorname{diag}\left(\mathbf{A}^{\prime}, \mathbf{A}^{\prime}, \mathbf{A}^{\prime}\right), \quad \mathbf{B}=\operatorname{diag}\left(\mathbf{B}^{\prime}, \mathbf{B}^{\prime}, \mathbf{B}^{\prime}\right), \quad \mathbf{C}=$ $\operatorname{diag}\left(\mathbf{C}^{\prime}, \mathbf{C}^{\prime}, \mathbf{C}^{\prime}\right), \mathbf{A}^{\prime}=\left[\begin{array}{ll}0 & 1 \\ 0 & 0\end{array}\right], \mathbf{B}^{\prime}=\left[\begin{array}{l}0 \\ 1\end{array}\right], \mathbf{C}^{\prime}=\left[\begin{array}{ll}1 & 0\end{array}\right]$. Thanks to the particular triangular form of (20), in order to get an estimation of $\mathbf{z}$, it is possible to apply the following nonlinear high gain observer (HGO) [7]

$$
\dot{\hat{\mathbf{z}}}=\mathbf{A} \hat{\mathbf{z}}+\mathbf{B} \boldsymbol{\sigma}\left(\hat{\mathbf{z}}, \mathbf{u}, \mathbf{W}_{R}, \mathbf{w}_{1}\right)+\mathbf{H}\left(\mathbf{w}_{\mathbf{z}}-\mathbf{C} \hat{\mathbf{z}}\right),
$$

where $\mathbf{H}=\operatorname{diag}\left(\mathbf{H}^{\prime}, \mathbf{H}^{\prime}, \mathbf{H}^{\prime}\right)$ and $\mathbf{H}^{\prime}=\left[\frac{\alpha_{1}}{\varepsilon} \frac{\alpha_{2}}{\varepsilon^{2}}\right]^{T}$, with $\varepsilon \in \mathbb{R}_{>0}$, and the gains $\left(\alpha_{1}, \alpha_{2}\right) \in \mathbb{R}_{>0}$ are set such that the roots of $s^{2}+\alpha_{1} s+\alpha_{2}$ have negative real part. Notice that the observer is made by a bank of three independent high gain observers of order two, the minimum order possible. This low order implies lower gains that in turn imply less peaking phenomenas and noise effects, which are instead typical in higher order HGO.

Summarizing, using the standard sensorial configuration of Tab. I, we obtained an estimation of the whole state:

$$
[\hat{l} \hat{i} \hat{\varphi} \hat{\dot{\varphi}} \hat{\delta} \hat{\delta}]^{T}=\hat{\mathbf{z}}, \quad \hat{\mathbf{R}}_{R}=\mathbf{W}_{R}, \quad \hat{\boldsymbol{\omega}}_{R}=\mathbf{w}_{6}
$$

\section{A. Closed Loop Stability}

In Sec. III we showed that the control law (16)-(18) needs only the knowledge of the state and of the trajectory of the platform in order to close the loop. Thus we can use the estimated state as feedback for the controller. Because the system is non-linear, one cannot apply the separation principle like in the linear case. Nevertheless, thanks to the particular triangular block dynamics with a direct measurement of the first state of each block, the conditions of [7, Theorem 14.6] holds. Therefore, since the closed loop system by state feedback is exponentially stable for every state except the zero thrust case, there exist a $\bar{\varepsilon}$ such that, for every $0<\varepsilon \leq \bar{\varepsilon}$ in (21), the closed loop system with the observer is exponentially stable, except for the zero thrust and zero tension case [7].

\section{B. Discussion on Measurement $\mathbf{w}_{1}$}

To obtain a perfect tracking (with any controller) one has to know the derivative of $\mathbf{p}_{C}^{C}(t)$ up to the fourth order and of $\boldsymbol{\omega}_{C}(t)$ up to the third order. Although in practice it is difficult to measure the higher-order derivatives, some practical techniques could be applied to overcome such issue. If the model and control input of the system are known (e.g., in the case of an autonomous vehicle), an observer can be designed to retrieve the needed derivatives of $\mathbf{p}_{C}^{C}$ and $\boldsymbol{\omega}_{C}$. Or if only a set of measurements of some derivatives is available, and

\footnotetext{
${ }^{1}$ For the startup one can, e.g., first tauten the cable using a near hovering control [10] and then switch to our controller to maintain the desired tension.
}

if the frequency content of the trajectory of the platform is sufficiently low, the unknown higher derivatives could be assumed negligible. For this last case, in Sec. V we show that in practice the tracking error remains small and bounded.

\section{Numerical VALIDATION}

In this section we provide some of the results obtained by simulation to test the validity of the proposed method ${ }^{2}$. We consider an aerial vehicle with mass $m_{R}=1[\mathrm{Kg}]$ and inertia $\mathbf{J}_{R}=\operatorname{diag}(0.25,0.25,0.25)\left[\mathrm{Kg} \mathrm{m}^{2}\right]$. We assume constant winch radius and inertia equal to $r_{W}=0.2[\mathrm{~m}]$ and $J_{W}=0.15\left[\mathrm{Kg} \mathrm{m}^{2}\right]$, respectively. We set $\mathbf{k}_{i}$ and $\mathbf{k}_{j}$ such that the error dynamics $\boldsymbol{\xi}_{i}$ and $\boldsymbol{\xi}_{j}$ have poles in $(-1,-2,-3,-4)$ and $(-1,-2)$ respectively. For the observer we choose $\varepsilon=0.1$ and $\left(\alpha_{1}, \alpha_{2}\right)$ such that $s^{2}+\alpha_{1} s+\alpha_{2}$ has roots $(-3,-4)$. Those values guarantee the stability and ensure a sufficiently fast exponential tracking.

Like in a real patrolling task, the platform follows a certain trajectory on the 3D space mimicking, e.g., a ground robot following a road. We require the aerial vehicle at time $t_{0}$ to takeoff from the moving platform, at time $t_{\text {circ }}$ to circle above the platform at a certain altitude, and at time $t_{\text {land }}$ to land on the moving platform (see Fig. 3c). The yaw angle of the aerial vehicle has to follow the one of the platform. During the takeoff the desired tension must go from a small initial tension of $0.5[\mathrm{~N}]$ to a steady-state value of $3[\mathrm{~N}]$, that is kept for the whole of the circling phase, and then has to go back to the initial value during the landing.

To fully validate our method for real applications we test the convergence and the robustness for different non-ideal cases commented in the following. Fig. 3 gathers the main results.

a) With an initial position and estimation error, after the convergence of the observer (less than one second) the outputs follow the desired trajectories with high fidelity.

b) With a parametric variation of $5 \%$ we notice a small constant error in the estimation of the state, but we obtain a monotonically decreasing tracking error thanks to the addition of an integral term in the outer loop (18), e.g., $v_{1}=y_{1}^{d(4)}+$ $\mathbf{k}_{1}^{T} \boldsymbol{\xi}_{1}+k_{I 1} \int_{0}^{t} \xi(\tau) d \tau$.

c) For a moving platform a standard sensorial set (e.g., optical flow, IMU and magnetometer) usually is sufficient to measure its trajectory variables up to $\ddot{\mathbf{p}}_{C}^{C}$ and $\omega_{C}$. Considering as zero the higher derivatives we observe that the estimation and tracking error is very small and remains always bounded under a reasonable threshold.

d) In the presence of Gaussian noise in the measurements with typical variance values, we notice that the state estimate becomes slightly noisy but the error remains bounded within small values. The non zero estimation error implies a non zero but bounded tracking error as well.

$e$ ) since in practice one cannot assume the link attached exactly to $O_{R}$, we tested the method for a vertical offset of $5[\mathrm{~cm}]$ with respect $\mathscr{F}_{R}$. In this case the tracking error does not go to zero but remains bounded below a small threshold.

f) Finally we implemented a controller based on a standard hierarchical method to compare it with our method. We noticed

\footnotetext{
${ }^{2}$ For the complete set of plots and several additional simulations in nonideal conditions we refer the interested reader to [12]. The reader is also encouraged to watch the accompanying video.
} 
that to obtain good tracking performances it requires very high gains that cause instability in the presence of the same noisy measurements of case d). Therefore we lowered the gains until we obtained a stable behavior. However these gains are not enough to obtain good tracking performances anymore. Moreover notice that the cable becomes even slack $\left(f_{L f}<0\right)$.

\section{CONCLUSIONS}

For the problem of an aerial vehicle tethered to an actuated winch, in turn attached to a generic moving platform, we proposed an accurate analysis of the intrinsic characteristics of the system and a novel nonlinear controller/observer scheme. The controller is able to steer the position of the aerial vehicle and the tension on the link along any smooth trajectories. This extends the previous works beyond the $2 \mathrm{D}$ plane and the simpler regulation control problem. To complete the control method we propose a nonlinear observer based on a set of standard sensors that is able to retrieve the state from any dynamic condition. Through exhaustive simulations (see [12]) we show the potentiality of the method for automated takeoff and landing from a moving platform, as well as for inspection and surveillance tasks, testing it under many non-ideal conditions.

In the future we plan to validate the method with real experiments in a plausible environment. Additional extensions could be, e.g., on planning for obstacle avoidance, or, in the case of an autonomous platform, on cooperative control.

\section{REFERENCES}

[1] M. Tognon and A. Franchi, "Nonlinear observer-based tracking control of link stress and elevation for a tethered aerial robot using inertial-only measurements," in 2015 IEEE Int. Conf. on Robotics and Automation, Seattle, WA, May 2015, pp. 3994-3999.

[2] S. Lupashin and R. D'Andrea, "Stabilization of a flying vehicle on a taut tether using inertial sensing," in 2013 IEEE/RSJ Int. Conf. on Intelligent Robots and Systems, Tokyo, Japan, Nov 2013, pp. 2432-2438.

[3] M. M. Nicotra, R. Naldi, and E. Garone, "Taut cable control of a tethered UAV," in 19th IFAC World Congress, Cape Town, South Africa, Aug. 2014, pp. 3190-3195.

[4] L. Sandino, D. Santamaria, M. Bejar, A. Viguria, K. Kondak, and A. Ollero, "Tether-guided landing of unmanned helicopters without GPS sensors," in 2014 IEEE Int. Conf. on Robotics and Automation, Hong Kong, China, May 2014, pp. 3096-3101.

[5] S.-R. Oh, K. Pathak, S. K. Agrawal, H. R. Pota, and M. Garrett, "Approaches for a tether-guided landing of an autonomous helicopter," IEEE Trans. on Robotics, vol. 22, no. 3, pp. 536-544, 2006.

[6] M. Tognon and A. Franchi, "Nonlinear observer for the control of bitethered multi aerial robots," in 2015 IEEE/RSJ Int. Conf. on Intelligent Robots and Systems, Hamburg, Germany, Sep. 2015, pp. 1852-1857.

[7] H. K. Khalil, Nonlinear Systems, 3rd ed. Prentice Hall, 2001.

[8] R. M. Murray, M. Rathinam, and W. Sluis, "Differential flatness of mechanical control systems: A catalog of prototype systems," in ASME Int. Mechanical Eng. Congress and Exposition, San Francisco, CA, Nov. 1995.

[9] P. Martin, P. Rouchon, and R. M. Murray, "Flat systems, equivalence and trajectory generation," Aug. 2006, lecture. [Online]. Available: https://cel.archives-ouvertes.fr/cel-00392180

[10] G. Gioioso, M. Ryll, D. Prattichizzo, H. H. Bülthoff, and A. Franchi, "Turning a near-hovering controlled quadrotor into a 3D force effector," in 2014 IEEE Int. Conf. on Robotics and Automation, Hong Kong, China, May. 2014, pp. 6278-6284.

[11] R. Mahony, T. Hamel, and J.-M. Pflimlin, "Nonlinear complementary filters on the special orthogonal group," IEEE Trans. on Automatic Control, vol. 53, no. 5, pp. 1203-1218, 2008.

[12] M. Tognon and A. Franchi, "Extended simulations for the observerbased control of position and tension for an aerial robot tethered to a moving platform,” LAAS-CNRS, Tech. Rep. hal-01261251, Jan. 2016. [Online]. Available: https://hal.archives-ouvertes.fr/hal-01261251

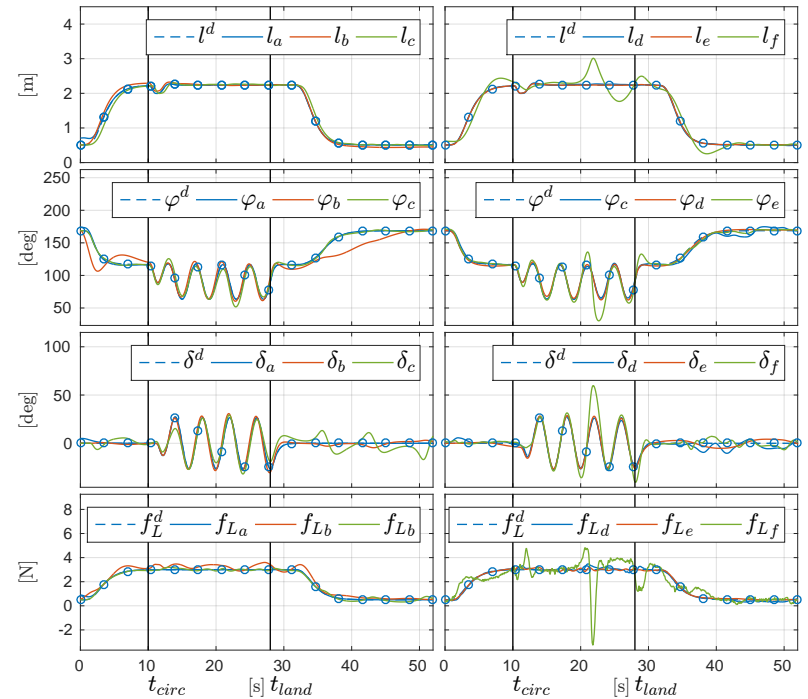

(a) Controller performances: the tracking of the output of interest for each case is plotted. The subscript of the variables indicates the relative case. The blue dots highlight the desired trajectory.

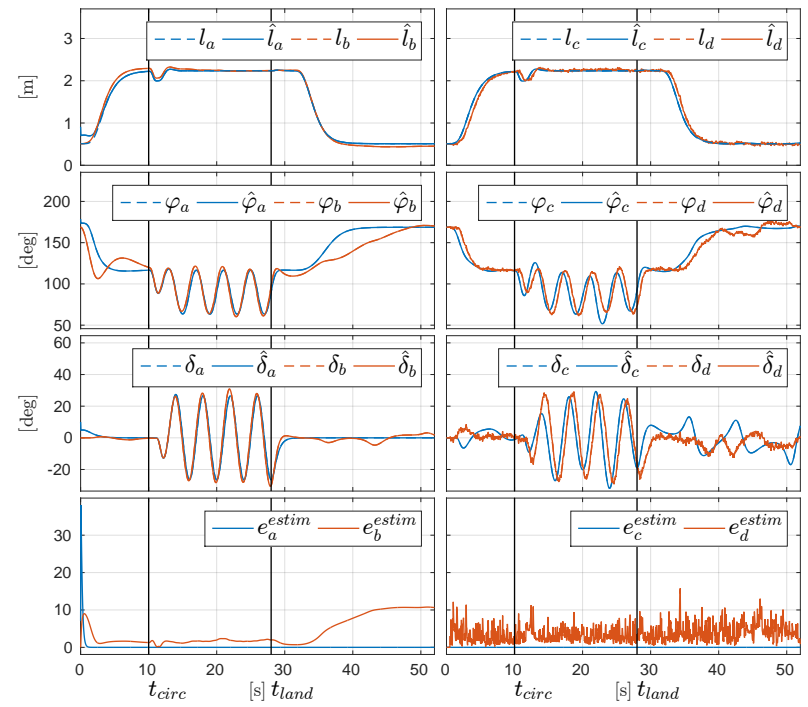

(b) Observer performances. The cases e) and f) are not plotted for space limitation. However they can be found in [12].

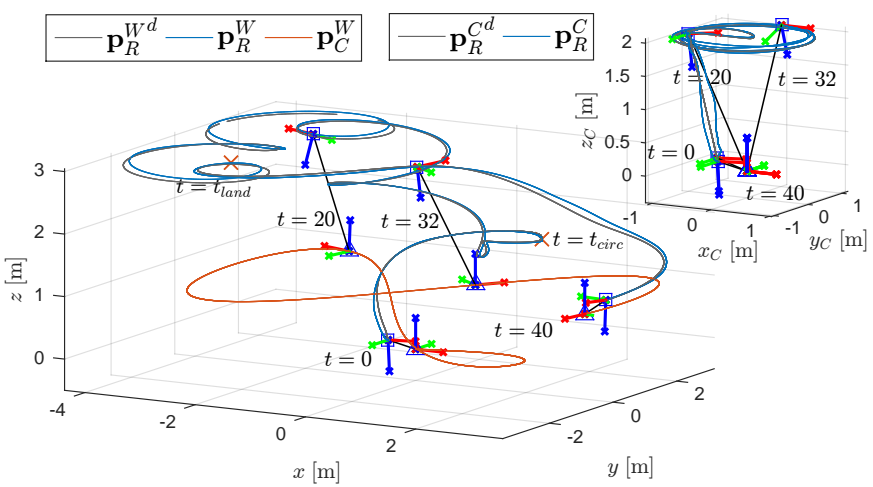

(c) Trajectories visualization for Case d). Left: trajectories in the world frame. Right: aerial vehicle trajectory in the moving platform frame.

Fig. 3: Simulation results of a plausible task trajectory. The performances for each non-ideal case are compared. Full plots and long explanations can be found in [12]. 